\title{
Innovaciones en la asignatura de Econometría para la Empresa en el Grado de Administración y Dirección de Empresas
}

\author{
ROSARIO ASIÁN CHAVES \\ UNIVERSIDAD DE SEVILLA \\ DPTO. ECONOMÍA APLICADA II \\ rasian@us.es \\ ORCID: https://orcid.org/0000-0001-6002-8900 \\ D.O.I.: http://dx.doi.org/10.12795/JDU.2018.i01.103 \\ Pp.: 1833-1853
}

\section{Resumen}

En esta comunicación se describe la aplicación de un ciclo de mejora en la asignatura de Econometría para la Empresa que tuvo lugar en la última semana de octubre y primera de noviembre de 2018. Se ha avanzado en algunos de los cambios propuestos, y a partir de los feedback con el alumnado y el análisis de los resultados de la evaluación he podido recapacitar acerca de la necesidad de continuar avanzando sobre esta base en el próximo curso.

Palabras clave: Econometría para la empresa, Experimentación Docente Universitaria, Metodología Docente, Enseñanza-aprendizaje 


\section{Breve descripción del contexto de la intervención}

La experiencia innovadora que se presenta en esta comunicación se realizó en la asignatura Econometría para la Empresa, obligatoria del 10 cuatrimestre de 30 curso del Grado en Administración y Dirección de Empresas (GADE), 6 créditos ECTS. Se aplicó en el grupo 4, que tiene horario de tarde: lunes y miércoles de 18:00 a 20:00.

La intención este curso ha sido ampliar lo ya iniciado el curso pasado, a partir del curso de iniciación. En principio, el curso pasado solo conseguí plantearme la necesidad de realizar cambios importantes en la metodología docente, que afectarían al contenido, forma de impartir las clases, evaluación... pero no avancé mucho. En este curso intentaré avanzar en el método de enseñanza-aprendizaje en esta asignatura, y en el ciclo de mejora he programado hacerlo paso por paso.

Uno de los objetivos planteados en el ciclo de mejora es conectar más la parte teórica y la práctica de esta asignatura, ya que los últimos años, sobre todo cuando se ha compartido docencia con otro profesor, los alumnos se han quejado de que parecen dos asignaturas diferentes. La aplicación el Ciclo de Mejora en el curso 2017-18, aunque solo conseguí incorporar algunos cambios en la metodología como he comentado al principio, funcionó bastante bien.

Este ciclo de mejora se ha basado, por tanto, en la experiencia del ciclo de mejora aplicado el curso pasado. El cuestionario de ideas previas se pasó el primer día de clase para que fuera justo antes de empezar a hablar de cualquier cuestión para que no influyese en sus respuestas. 


\section{Diseño del CM}

\section{Mapa de contenidos y problemas o preguntas-clave.}

El CMD está basado en un caso concreto: "Vamos a plantearnos que queremos montar un negocio en mi pueblo, por ejemplo una tienda de bicicletas, y lo primero que me pregunto es de qué va a depender mi volumen de ventas, es decir, qué variables van a influir en que venda más o menos y cómo las voy a relacionar y de qué manera y hasta dónde ello me puede servir para mejorar mis resultados". El objetivo es que el alumnado sea consciente de qué puede conseguir con el conocimiento y aplicación de esta asignatura en el contexto real y profesional.

Los contenidos conceptuales y procedimentales trabajados son los siguientes:

Jornadas de Formación e Innovación Docente del Profesorado | № 1 (2018) Esta obra se distribuye con la licencia Creative Commons 


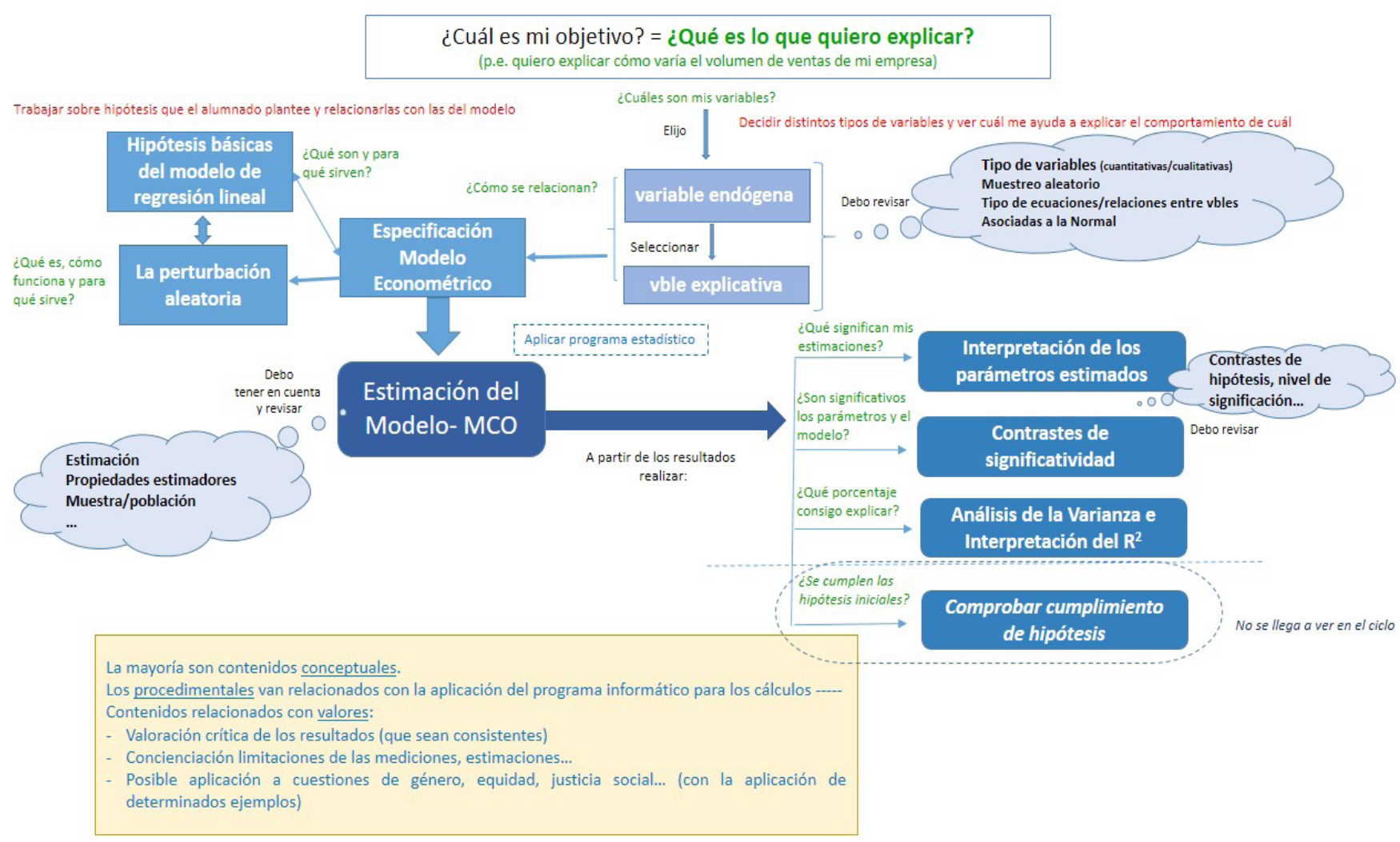

Figura 1. Mapa de contenidos del tema de Estimación del Modelo por MCO

Jornadas de Formación e Innovación Docente del Profesorado | № 1 (2018)

(c) $(1)(2)$ Esta obra se distribuye con la licencia Creative Commons

Internacional (CC BY-NC-ND 4.0.) 
1. Selección de las variables del modelo; 2. Especificación del modelo econométrico; 3. Estimación del modelo por el método de mínimos cuadrados; 4. Utilización de software para la estimación. (PROCEDIMENTAL); 5. Interpretación de las estimaciones de los parámetros del modelo; 6. Contrates de significatividad; 7. Análisis de la varianza e interpretación del R²; 8. Hipótesis del modelo; 9. Validación (primera parte).

Los tres primeros van dirigidos fundamentalmente a abordar el objetivo o finalidad acerca de que el alumnado adquiera un conocimiento básico de los problemas de especificación. Para ello, si bien muchos de los contenidos son conceptuales: tipos de variables, requisitos para la correcta especificación del modelo, concepto de estimador/ estimación, características deseables en los estimadores, etc., requieren para su aplicación de contenidos procedimentales: aplicación del programa informático para estimar el modelo, por ejemplo. Con estos contenidos procedimentales se aborda la finalidad de que el alumnado tenga las habilidades necesarias para poder aplicar software de diversos métodos econométricos sobre conjuntos seleccionados de datos.

Una vez estimado el modelo hay que pasar a la interpretación de resultados (punto 5), que requiere además comprobar si son consistentes y válidos para explicar lo que nos hemos planteado como objetivo último, para lo cual necesitaré realizar contrastes de hipótesis sobre la significatividad del modelo y de las estimaciones de mis parámetros (punto 6) y comprobar qué porcentaje de lo que quiero explicar lo puedo conseguir con el modelo utilizado (punto 7).

El punto 8 combina una serie de contenidos conceptuales: qué es una hipótesis nula, qué es la región crítica de un contraste, análisis de la varianza, etc., procedimentales: cómo se calcula el estadístico que permite construir la región crítica, o el p-valor del contraste y cómo se interpretan; cómo obtenerlo en el programa informático que utilizamos...; y contenidos basados en valores: lo que hemos planteado como valoración crítica de resultados numéricos 
(preguntarme si el resultado es consistente y lógico, así como sus limitaciones, es decir ¿hasta dónde me sirven para explicar la realidad?), conocer y ser conscientes de las limitaciones de las mediciones y de las estimaciones, y trabajar valores relacionados con cuestiones de género, equidad, justicia social, etc. Esto lo planteamos a partir de determinados ejemplos donde se ponen de manifiesto algunas diferencias que sirven para explicarlos, por ejemplo en las diferencias salariales entre hombres y mujeres, entre trabajadores de distinta raza, nacionales o extranjeros, diferente estatus social, etc.

En realidad, en las 8 horas para las que he planteado el ciclo de mejora, no ha dado tiempo de abordarlo todo, pero he tratado de hacerlo en la mayor medida de mis y sus posibilidades, para en cursos posteriores poder seguir avanzando. Por ello, he tratado de centrar mi mapa de contenidos a lo que ha sido posible abarcar en esas 8 horas.

En el mapa de contenidos (figura 1) quedan reflejados tanto los contenidos conceptuales (variables, especificación del modelo, etc.), procedimentales (aplicación del programa informático para la estimación del modelo y obtención de principales resultados, así como para la realización de ciertos contrastes) y en el recuadro inferior derecho se incluyen los relacionados con valores. Se han incluido en verde las preguntas clave que se tratan de responder en cada explicación de cada concepto.

En las nubes celestes se han incluido aquellos contenidos que se deben revisar, pero que ya se han explicado (en los temas introductorios o en otras asignaturas). La parte en que se profundiza más es en la estimación del modelo por Mínimos Cuadrados Ordinarios (MCO) y en la interpretación de los resultados obtenidos: Interpretación parámetros estimados, contrastes de significatividad, análisis de la varianza e interpretación del R2 (todo ello en la parte derecha del mapa). Las hipótesis se ven es este tema e insistimos en la necesidad de su cumplimiento y en su significado en el modelo, pero los contrastes específicos 
para contrastar su cumplimiento y las medidas correctoras se tratarán en temas posteriores. Igualmente, la finalidad del modelo (predicción o toma de decisiones), solo se perfilan, pero se tratarán más detenidamente en otros temas del programa.

En las sesiones en las que se ha desarrollado el ciclo de mejora se ha tratado de responder a la siguiente pregunta:

¿Cómo puedo explicar la variabilidad de una variable seleccionada, por ejemplo el volumen de ventas de mi empresa? Para ello, debo responder a:

- ¿Qué variable puedo seleccionar como la que mejor me sirva para responder a la pregunta anterior?

- ¿Qué relación creo que existe entre esas variables? ¿Cómo puedo tener en cuenta el resto de variables que también me están influyendo?

- ¿Cómo y dónde puedo obtener los datos que necesito para cuantificar mis variables?

- ¿De qué manera puedo traducir todo ello en un modelo que me sirva para estimar aquello que deseo explicar?

- ¿Cómo puedo interpretar los resultados obtenidos?

\section{Modelo metodológico y Secuencia de Actividades.}

El modelo metodológico usado habitualmente consistía en un modelo basado en la metodología tradicional de exposición de los contenidos (clase magistral) para que el alumnado lo trabaje por su cuenta. Se sigue la secuencia de Bienvenida/presentación, Recapitulación/Introducción, Desarrollo teórico o práctico de la clase y Resumen/ adelanto:

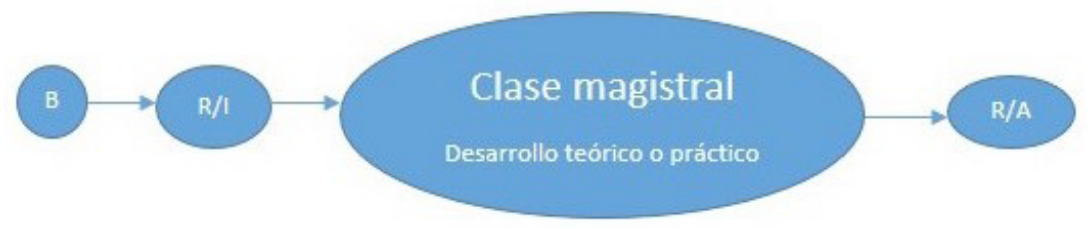

Jornadas de Formación e Innovación Docente del Profesorado | № 1 (2018) Esta obra se distribuye con la licencia Creative Commons Reconocimiento-NoComercial-SinObraDerivada Internacional (CC BY-NC-ND 4.0.) 
En el modelo ideal me planteo comenzar todas las clases a partir de la resolución de un problema o pregunta. Previamente, se pasaría un cuestionario inicial que después se compararía con el cuestionario final para evaluar la evolución del aprendizaje. El material sería estudiado o analizado previamente por el alumnado a partir de lo que les suba a la plataforma y con lo que investiguen y busquen por su cuenta, y en las clases solo se recurriría a hacer alguna explicación cuando planteen alguna duda sobre lo que han trabajado o cuando yo viese que algo parece que no les ha quedado suficientemente claro. Tendría que diseñar unas actividades de contraste para desarrollar a lo largo del proceso.

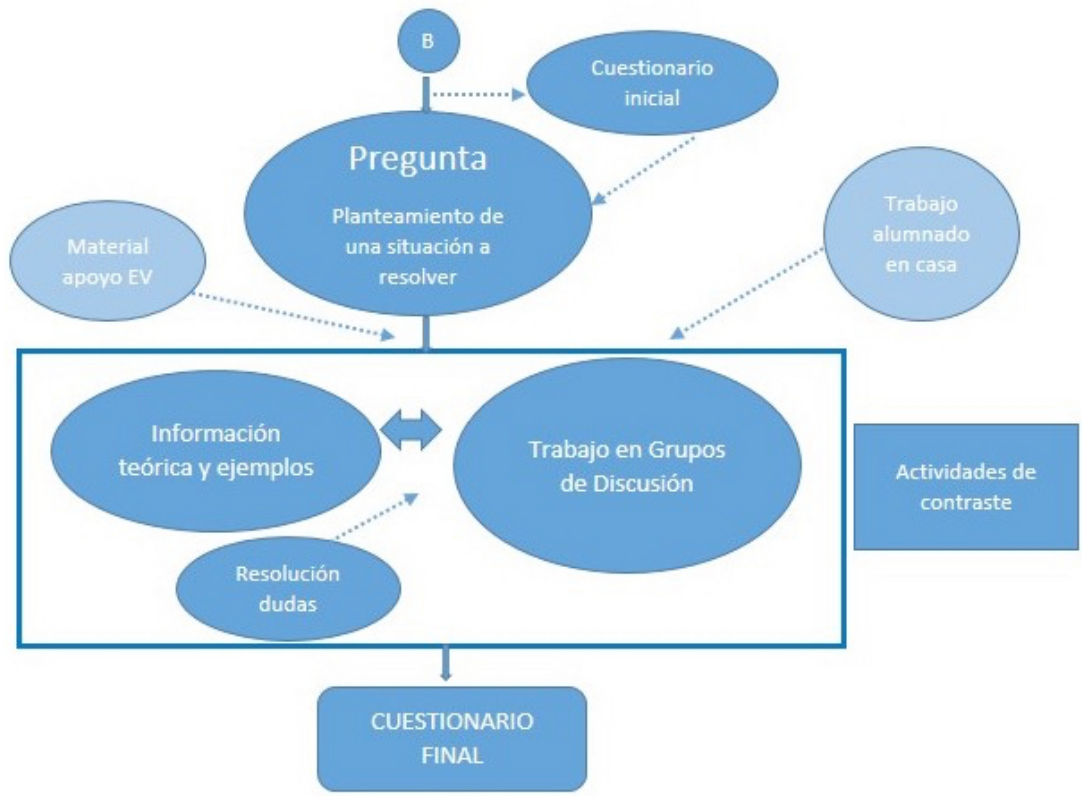

Mientras consigo llegar a ese modelo ideal, en el que mi intervención en clase sería de observadora y guía del trabajo del alumnado, que es quien actúa y es el verdadero protagonista en la clase, tengo que seguir trabajando en mis ciclos de mejora, por lo que para este curso me planteo el modelo posible que explico a continuación. 
El modelo posible en este momento: Mi propuesta consiste en acercar mi metodología lo más posible a la estrategia didáctica del "Aprendizaje Basado en problemas" (ABP), aunque no se puede considerar del todo un ABP, sino una mezcla entre varios métodos, según el caso:

- Método expositivo/lección magistral: para introducir ciertos conceptos y a veces para explicar algún procedimiento.

- Estudio de casos simulados, a partir de los datos que utilizamos y los modelos que formulamos como si fuese para un caso real.

- Una mezcla entre Resolución de ejercicios y problemas y ABP.

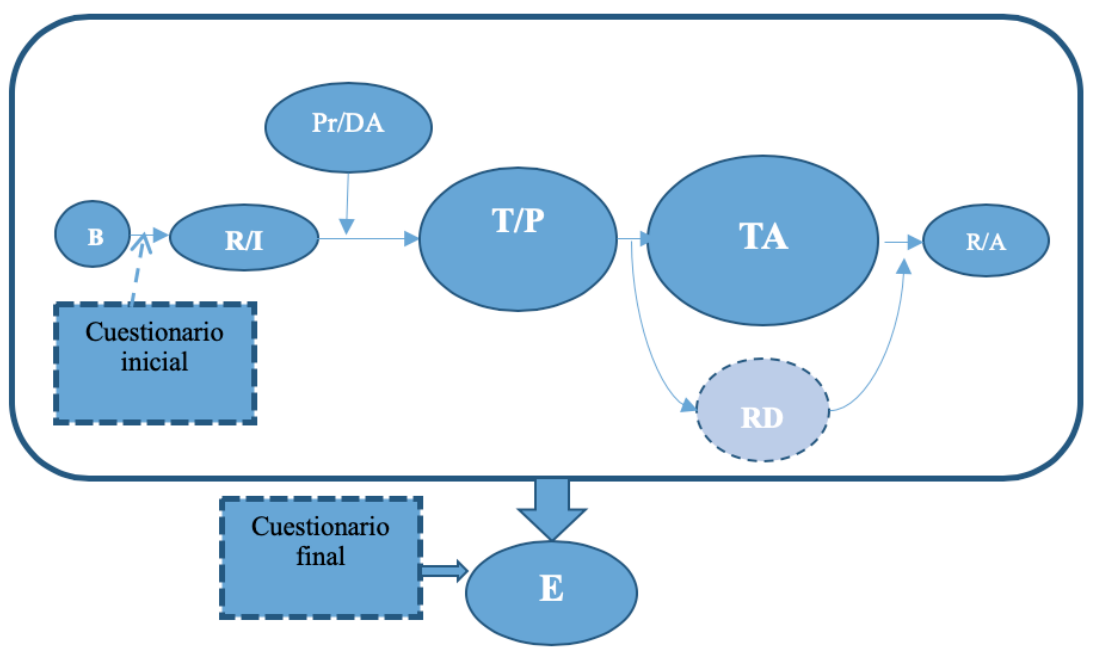

B: bienvenida, saludo

$\mathrm{R} / \mathrm{I}$ : revisión/introducción

Pr/DA: Pregunta-problema/Discusión-Debate Alumnos T/P: Explicación teoría y aplicación a una práctica

TA: Trabajo alumnado sobre su propio modelo RD: Resolución de dudas a lo largo del TA $\mathrm{R} / \mathrm{A}$ : revisión/avance

Lo hemos visto para la quinta y sexta semana del curso, cuando ya se ha introducido brevemente en qué consiste 
la asignatura y algunos conceptos fundamentales, y se ha hecho hincapié en el repaso de algunas nociones de Estadística que son fundamentales para la Econometría, cuando estábamos terminando el tema 2 del programa: "Construcción de un modelo Econométrico. El caso de una variable explicativa".

\section{SECUENCIA DE ACTIVIDADES}

Las dos semanas primeras de clase, previas al inicio del ciclo de mejora, he intentado que vean los tipos de variables con las que podemos trabajar, que repasen ciertos conceptos estadísticos básicos (distribución Normal y sus asociadas, estimación, inferencia, etc.) y visualizar de manera global el contenido de la asignatura y empezar con los tipos de modelos y las hipótesis básicas, para ya en la tercera semana empezar a abordar el tema 2. Como este tema es muy amplio, cubre gran parte de la esencia de la asignatura, hemos centrado el ciclo de mejora a partir de la quinta semana que ya están más familiarizados con el vocabulario, la técnica.... En la primera clase del curso, antes del inicio del ciclo de mejora, pasé tres tipos de cuestionarios breves al alumnado:

1. Sobre sus expectativas, ideas, etc. sobre la asignatura, motivos de elección del grupo (ya que son 6 grupos), ...

2. Sobre algunos conocimientos que se supone deben tener de Estadística

3. Sobre ideas previas de lo que vamos a ver en el tema 2 correspondiente a nuestro ciclo de mejora.

En las dos semanas del ciclo, la secuencia ha sido la siguiente:

Jornadas de Formación e Innovación Docente del Profesorado I № 1 (2018) Esta obra se distribuye con la licencia Creative Commons 
Tabla 1. Secuencia de actividades

\begin{tabular}{|l|l|}
\hline Actividad & Tiempo \\
\hline B: bienvenida, saludo & 5 minutos \\
\hline R/I: revisión/introducción & 10 minutos \\
\hline $\begin{array}{l}\text { Pr/DA: Preguntas-problemas / Discusión-Debate } \\
\text { Alumnos }\end{array}$ & $10-15$ minutos \\
\hline $\begin{array}{l}\text { T/P: Explicación teoría y aplicación a una práctica } \\
\text { con ejemplo diseño }\end{array}$ & $40(20)$ minutos \\
\hline TA: Trabajo alumnado sobre su propio modelo & $20(40)$ minutos \\
\hline RD: Resolución de dudas a lo largo del TA & $10(20)$ minutos \\
\hline $\begin{array}{l}\text { R/A: Revisión Teórica-Práctica conceptos, procedi- } \\
\text { mientos y valores /avance }\end{array}$ & 5 minutos \\
\hline E: Evaluación* & $(40+5$ minutos) \\
\hline última Sesión O clase &
\end{tabular}

\section{Cuestionario inicial-final para hacer seguimiento de evolución}

Como se ha comentado al describir la secuencia de actividades, se han diseñado unos cuestionarios iniciales para analizar el punto de partida del alumnado y su nivel de conocimiento sobre algunos de los contenidos a impartir. Asimismo, se ha pasado el mismo cuestionario al final del ciclo para comprobar el grado de aprendizaje adquirido, y poder observar el escalón en el que se encuentran, para poder determinar las necesidades y pensar qué aspectos pueden bloquear el paso a niveles superiores en la escalera. Este cuestionario ha sido el siguiente:

"Imagina que decides abrir un negocio en tu pueblo, por ejemplo una tienda de bicicletas. Intenta responder las siguientes preguntas:

1) Si quieres determinar el volumen de ventas, ¿de qué variables crees que va a depender?

2) Selecciona solo una de ellas, ¿Se podría plantear un modelo que pueda explicar la variabilidad del volumen de ventas $(Y)$ en función de la variable elegida $(X)$, o faltaría algo? En caso de faltar algo, ¿cómo lo podrías resolver?

3) ¿Qué tipo de datos necesitas? ¿De dónde los obtendrías?

Jornadas de Formación e Innovación Docente del Profesorado | № 1 (2018) Esta obra se distribuye con la licencia Creative Commons 
4) Si el modelo para plantear la explicación de la variabilidad en el volumen de ventas fuese $Y_{i}=\beta_{0}+\beta_{1} X_{i}+\varepsilon_{i}$, ¿qué crees que representa $\varepsilon_{i}$ ? ¿Para qué sirve?

5) En el modelo anterior, ¿Cuál sería la interpretación de los coeficientes $\beta_{0}$ y $\beta_{1}$ ?

6) ¿Y cuáles cree que serían sus signos? ¿Por qué?

7) ¿Cómo crees que se estimarían los valores de dichos coeficientes?

8) Con la información obtenida de una muestra de 10 observaciones se ha estimado el modelo $Y_{i}=4+0{ }^{\prime} 5 X_{i}+e_{i}$. Sabemos que la suma de los valores muestrales de $X$ es 160, ¿Cuál es el valor medio de los valores muestrales de la variable Y? ¿Por qué?

\section{Aplicación del CM}

\section{Relato Resumido de las sesiones.}

En las dos primeras sesiones, aunque siguiendo una dinámica similar a la que se ve en la secuencia de actividades, la mayor parte de esas dos sesiones son más parecidas a clases magistrales, aunque todo el tiempo parando y preguntando y dando lugar a la intervención del alumnado. Lo planteo así para poder avanzar los conceptos y así poder dedicar más tiempo en las sesiones 3 y 4 a que el trabajo del alumnado ocupe más tiempo y a su vez sea más productivo.

\section{Sesión 3.}

Actividad 1: Antes de comenzar, tras la bienvenida y saludos (5 minutos), hacemos un breve repaso de los conceptos vistos los primeros días (tipos de variables y datos, tipos de ecuaciones, concepto de regresión/correlación, fases en la especificación del modelo,...) para introducir la explicación de lo que vamos a ver en la clase de ese día (10 minutos). Recordamos algunas cuestiones analizadas en el tema 1, y primeros apartados del tema 2: las fases para la construcción del modelo para el modelo con una sola variable explicativa. Insistimos en la especificación (que ya 
está explicada, pero que ahora debe considerar la relación entre la variable explicativa, y la correcta selección de la más conveniente).

Actividad 2: A partir del planteamiento de algunas preguntas clave, y con su reflexión sobre el cuestionario que les pasé el primer día de clase, comienzan a discutir en grupos de 3a alumnos/as sobre sus planteamientos. Yo voy a provechando para escucharles e ir comentando, o en el grupo, o al conjunto de la clase cuando son temas de interés general. (15 minutos)

Actividad 3: A partir de aquí, comienzo con la continuación de la explicación teórica del tema (20-40 minutos) para que una vez definido y detallado de manera teórica lo podamos aplicar a los distintos tipos de modelos que han elegido previamente los grupos (10 minutos). Comprobamos cómo queda en cada modelo y comparamos los resultados (10 minutos). En esta sesión les recuerdo que para práctica deben ponerse de acuerdo para en sus grupos de tres personas y buscar unos datos con los que trabajar en la clase de práctica del próximo día.

Actividad 4: Antes de dar por finalizada la clase dedicamos 10 minutos a hacer una recopilación de lo visto en la clase, para hacer hincapié y resaltar aquellos aspectos fundamentales sobre los que tendrán que prestar especial atención cuando estudien la materia que se ha visto ese día. A continuación, los últimos 5 minutos les adelanto lo que vamos a ver el siguiente día para que se hagan una idea de lo que tenemos que ver para que decidan si lo quieren revisar antes (ya que disponen de todos los apuntes en la plataforma), o si consideran que tienen que repasar algún concepto o procedimiento que vieron el curso pasado en Estadística y que van a necesitar recordar para poder seguir sin dificultad la siguiente clase.

\section{Sesión 4.}

En esta clase vamos al aula de informática para trabajar en la aplicación práctica de lo que vimos en la sección más teórica. El esquema es muy similar al anterior: 
Actividad 1: Nuevamente comenzamos con la bienvenida y saludos (5 minutos). En este caso hacemos un breve repaso/revisión de los conceptos vistos en la clase de teoría que vamos a aplicar hoy concretamente a la especificación de nuestro modelo y su estimación (10 minutos).

Actividad 2: La siguiente media hora de clase la dedicamos a aplicar los procedimientos de especificación y estimación del modelo, según lo visto en teoría, y sobre un ejercicio/caso que yo traigo preparado y que voy desarrollando en mi ordenador y ellos pueden ir viendo a través de su proyección en la pantalla y pueden ir realizando a su vez en sus propios ordenadores (20 minutos).

Actividad 3: Una vez han visto cómo se debe proceder, les dejo para que empiecen a aplicarlo sobre sus propios datos que deben traer ya buscados para empezar a trabajar con ellos. Ahora yo voy pasando por toda la clase para ver qué van haciendo y resolver las distintas dudas que les van surgiendo a medida que van desarrollando los procedimientos antes realizados pero que ahora aplican a sus propios datos y que por tanto les dan resultados diferentes que a veces les cuesta interpretar (45 minutos).

Actividad 4: A continuación, se plantea la realización de nuevo del cuestionario que realizaron como evaluación inicial o de conocimientos previos en el primer día de clase. Ahora por grupos ponen en común sus respuestas iniciales y discuten cómo las modificarían con lo que ya han visto en estas sesiones que hemos tenido después del cuestionario (10 minutos). Les dejo otros 10 minutos para su realización individual, ya que la evaluación es individual aunque lo hayan podido discutir en grupo.

Actividad 5: A partir de aquí, recapitulamos y les resuelvo dudas y les planteo o reviso aquellas cuestiones que parece que puedan no quedar tan claras. (10 minutos) Actividad 6: Para finalizar, les paso otro cuestionario para que en 5-10 minutos evalúen el desarrollo de estas 4 sesiones pertenecientes al ciclo de mejora (a mi incluida) y realicen sus propuestas de mejora. 
Evaluación del aprendizaje de los estudiantes.

Para evaluar el aprendizaje del alumnado voy a basarme en las escaleras de aprendizaje. Para ello, de las 8 preguntas realizadas en el cuestionario inicial voy a analizar las cuestiones 4, 5 y 6. Primero presento la tabla con las respuestas agrupadas en bloques y número de alumnos/ as en cada caso para el cálculo de los porcentajes y a continuación las escaleras inicial y final.

Solo he tomado las respuestas de los 35 alumnos/as que respondieron ambos, porque hay varios que estuvieron el primer día y no vinieron al último, y sobre todo, hay muchos que respondieron el último día pero que el primer día no habían venido a clase porque a principio de curso todavía no tienen claro cuál va a ser su grupo definitivo.

\section{4) Si el modelo para plantear la explicación de la va- riabilidad en el volumen de ventas fuese $Y i=\beta 0+\beta 1 X i+\varepsilon i$, ¿qué crees que representa ci?? ¿Para qué sirve?}

\begin{tabular}{|l|l|c|c|c|c|}
\hline \multicolumn{2}{|l|}{ Respuesta } & $\begin{array}{l}\text { No } \\
\text { responde }\end{array}$ & $\begin{array}{l}\text { No sabe qué re- } \\
\text { presenta pero } \\
\text { intuye más 0 } \\
\text { menos para qué } \\
\text { puede servir }\end{array}$ & $\begin{array}{l}\text { Indica el } \\
\text { nombre } \\
\text { de } \varepsilon_{\boldsymbol{i}} \text { y se } \\
\text { acerca a su } \\
\text { función }\end{array}$ & $\begin{array}{l}\text { Indica el } \\
\text { nombre de } \\
\varepsilon_{\boldsymbol{i}} \text { y sabe } \\
\text { para qué } \\
\text { sirve }\end{array}$ \\
\hline Inicial & $\begin{array}{l}\text { No } \\
\text { alumnos }\end{array}$ & 9 & 23 & 3 & 0 \\
\hline Final & Porcentaje & $25,7 \%$ & $65,7 \%$ & $8,6 \%$ & $0,0 \%$ \\
\hline & $\begin{array}{l}\text { No } \\
\text { alumnos }\end{array}$ & 6 & 12 & 13 & 4 \\
\hline & Porcentaje & $17,2 \%$ & $34,3 \%$ & $37,1 \%$ & $11,4 \%$ \\
\hline
\end{tabular}

ESCALERA INICIAL

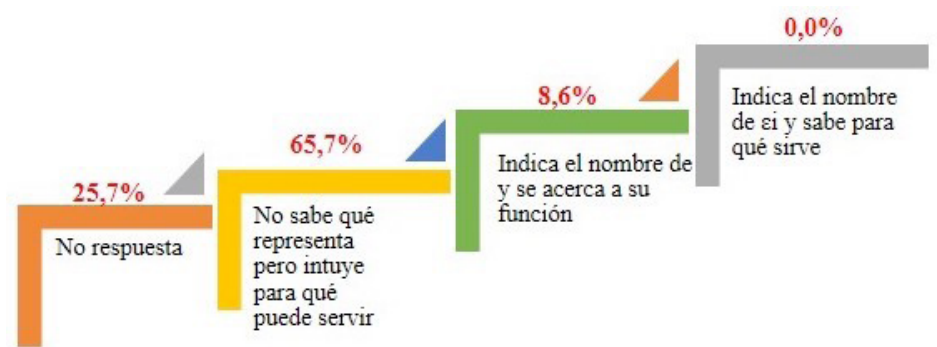

Jornadas de Formación e Innovación Docente del Profesorado | № 1 (2018)

Esta obra se distribuye con la licencia Creative Commons Reconocimiento-NoComercial-SinObraDerivada $\quad 4.0$ Internacional (CC BY-NC-ND 4.0.) 
ESCALERA FINAL

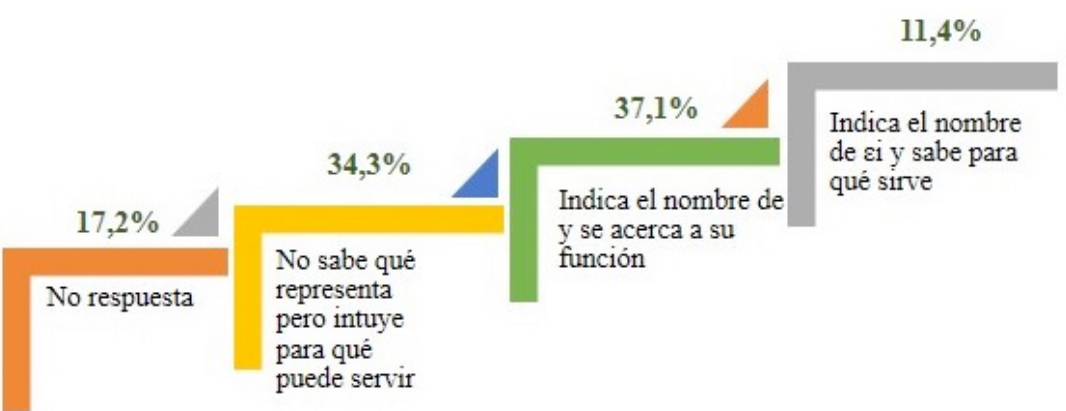

En estas primeras preguntas, la mayoría debía al menos intuir para qué podría servir. Al pasar el cuestionario nuevamente tras haberlo explicado en clase, como es algo bastante fácil, la mayoría debería ser capaz ahora de responder todo correctamente, pero se sigue manteniendo cierta dificultad, aunque realmente muchos de los que han respondido al final se han incorporado tarde al curso por lo que es posteriormente cuando han empezado a ver muchas de estas cosas que son del inicio.

5) En el modelo anterior, ¿cuál sería la interpretación
de los coeficientes $\boldsymbol{\beta}_{\boldsymbol{~}} \mathbf{y} \boldsymbol{\beta}_{\mathbf{1}}$ ?
\begin{tabular}{|l|l|c|c|c|c|}
\hline \multicolumn{2}{|l|}{ Respuesta } & $\begin{array}{l}\text { No } \\
\text { responde }\end{array}$ & $\begin{array}{l}\text { Ponen algo } \\
\text { que no tiene } \\
\text { nada que ver }\end{array}$ & $\begin{array}{l}\text { Se aproximan } \\
\text { levemente a la } \\
\text { interpretación }\end{array}$ & $\begin{array}{l}\text { Lo hace } \\
\text { correctamente }\end{array}$ \\
\hline Inicial & № alumnos & 7 & 24 & 4 & 0 \\
\hline & Porcentaje & $2,0 \%$ & $68,6 \%$ & $11,4 \%$ & $0,0 \%$ \\
\hline Final & № alumnos & 3 & 19 & 10 & 3 \\
\hline & Porcentaje & $8,6 \%$ & $54,2 \%$ & $28,6 \%$ & $8,6 \%$ \\
\hline
\end{tabular}

ESCALERA INICIAL

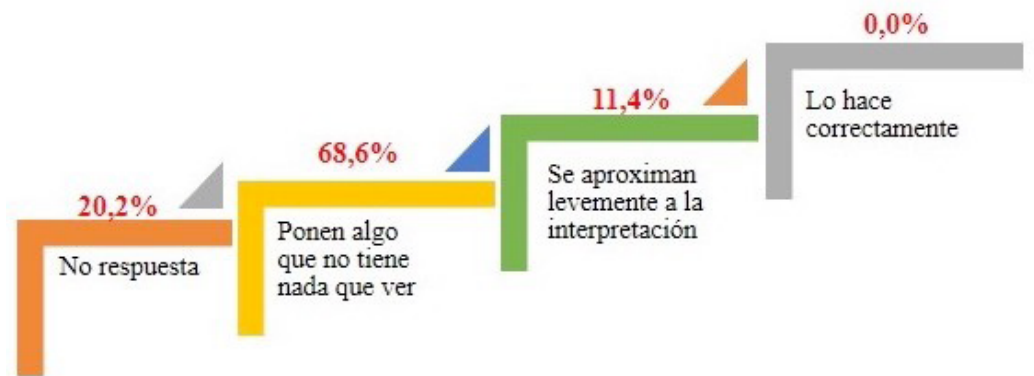

Jornadas de Formación e Innovación Docente del Profesorado | № 1 (2018)

Esta obra se distribuye con la licencia Creative Commons Reconocimiento-NoComercial-SinObraDerivada $\quad 4.0$ Internacional (CC BY-NC-ND 4.0.) 
ESCALERA FINAL

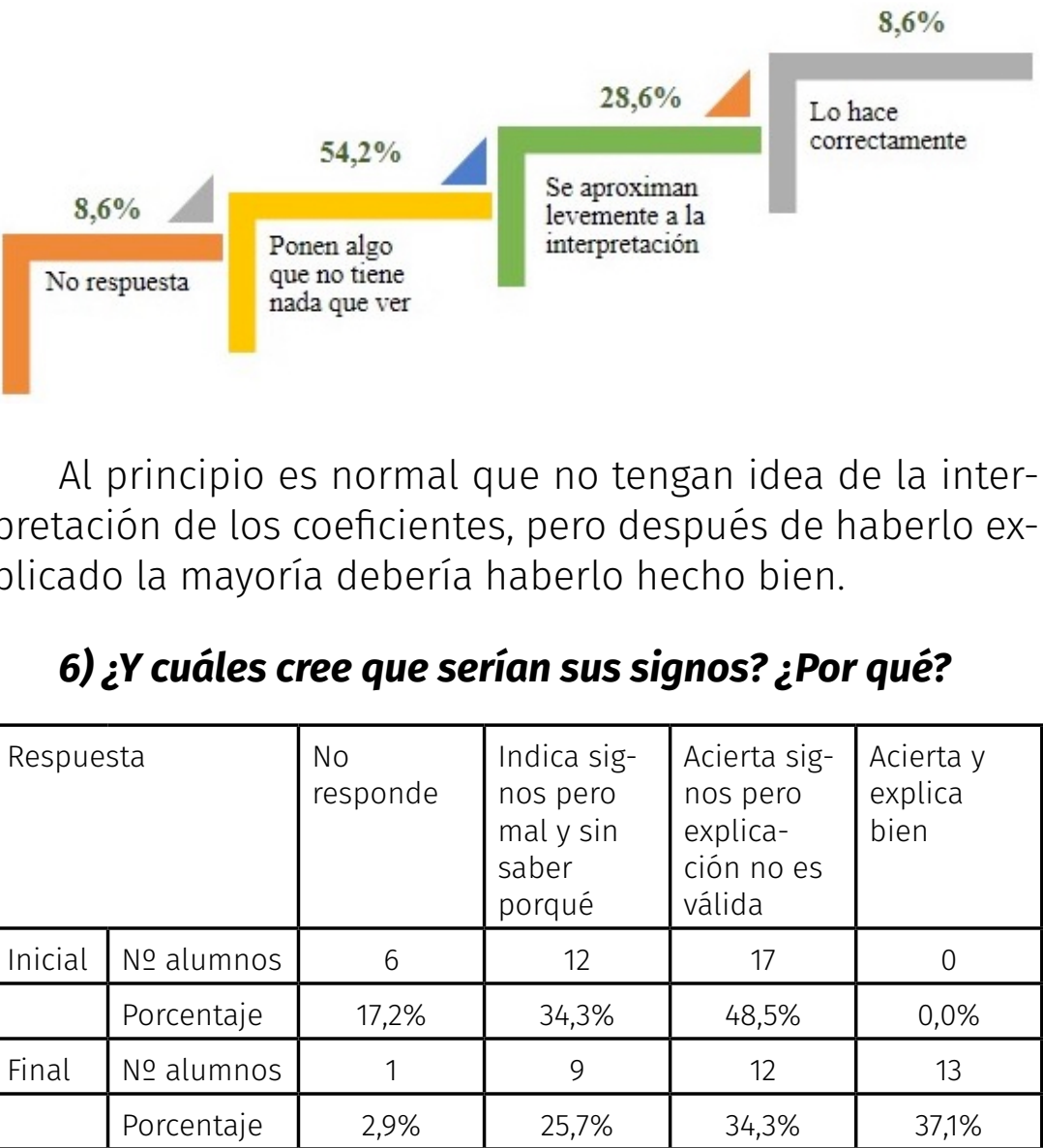

ESCALERA INICIAL

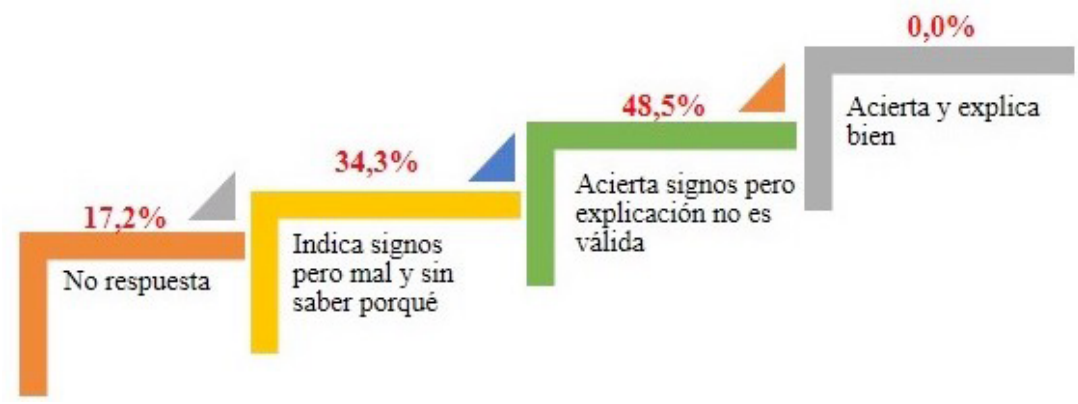

Jornadas de Formación e Innovación Docente del Profesorado | № 1 (2018)

Esta obra se distribuye con la licencia Creative Commons Reconocimiento-NoComercial-SinObraDerivada $\quad 4.0$ Internacional (CC BY-NC-ND 4.0.) 
ESCALERA FINAL

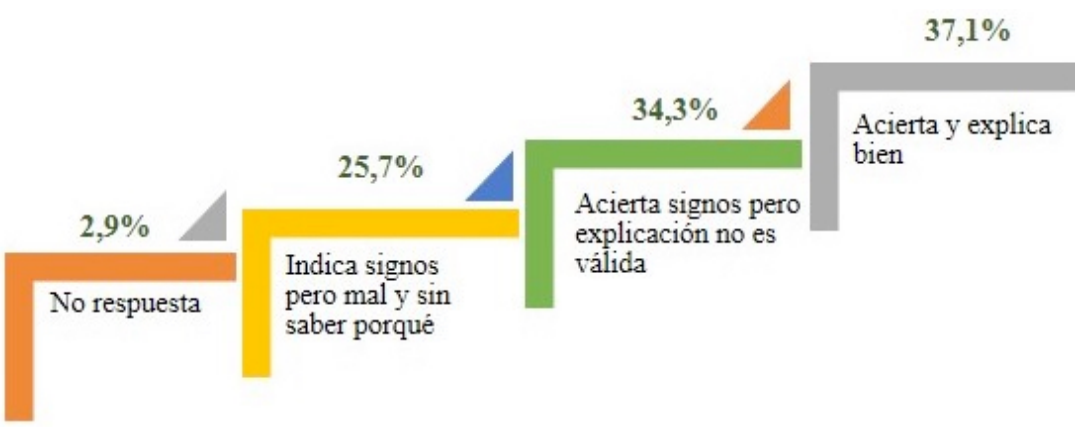

Podrían intuir, aunque no supiesen explicarlo, los signos de los coeficientes. Después de explicarlo en clase, más de la tercera parte supo explicar correctamente el motivo del signo que se espera tengan los parámetros, aunque todavía hubo un conjunto de alumnos/as que no supieron interpretarlo.

En general, el análisis de estas escaleras me lleva a plantearme la necesidad de realizar algunos cambios. También, por otro lado, que el cuestionario inicial si se hace tan al principio hay mucho alumnado que no coincide en los primeros días porque después se van incorporando al grupo así como otros dejan de venir. Es mejor hacerlo con el curso un poco más avanzado. También tengo que pensar cómo motivarles mejor para que traten de esforzarse en responder aunque no les vaya a servir para la calificación.

\section{Evaluación del diseño puesto en práctica}

\section{Cuestiones a mantener y cambios a introducir.}

Tanto la realización del curso de formación inicial del profesorado que realicé el año pasado, como sobre todo la continuación a través de la REFID y las reuniones mantenidas con mis compañeras de grupo han servido para estar más preparada y sentirme más segura a la hora de poner 
en marcha determinadas acciones. Sin embargo, dada la complejidad del proceso y que la evolución y resultados dependen de numerosos factores, esto ha hecho que algunas acciones no hayan tenido los resultados esperados. Por un lado creo que me falta plantearme con más tranquilidad los momentos de realización de las actividades y calcular mejor los tiempos de trabajo de cada tarea.

En esta fase creo que ha habido actividades para las que hubiera requerido más tiempo de discusión por mi parte con ellos y de maduración por parte del alumnado. Además, creo que estos cambios requieren de una mayor implicación por parte del alumnado, por lo que se requeriría un mayor esfuerzo por vincular los conocimientos a sus inquietudes e intereses, y la necesidad motivarles para que hagan un esfuerzo en actividades que no son evaluables directamente en su nota final.

En la evaluación a través delos cuestionarios iniciales y finales me reitero en la necesidad de realizarlo más adelante y no tan al principio, pues están sesgados dado el número de alumnos/as que no estaban al principio y que han tenido mejores resultados en el último cuestionario pero no he podido utilizar al no tener hechos los iniciales.

\section{Aspectos de la experiencia a incorporar en la práctica docente habitual}

Me gustaría poder continuar con el diseño metodológico planteado. Mi intención es modificar algunos de los contenidos y extraer los principales, para poder llevar a cabo una docencia participativa. En este sentido es necesario que pueda coordinarme con los compañeros/as de asignatura pues es muy complicado utilizar una metodología y un abordaje diferente en los distintos grupos de la misma asignatura en los que imparto docencia, y al compartir la mayoría de grupos, en los que bien imparto teoría o bien práctica y otro compañero la otra parte, no puedo 
utilizar los cambios planteados sin que la otra persona esté de acuerdo.

\section{Principios didácticos argumentados.}

En los siguientes cursos mi planteamiento ideal sería desarrollar lo siguiente:

- Lo primero y principal es tratar de acordar lo máximo posible con el resto de compañeros/as de asignatura el contenido, la metodología y, fundamentalmente, el método de evaluación, para no llevar a confusión y/o malestar al alumnado.

- Antes de comenzar las clases construir mapas conceptuales y organizar las secuencias de actividades de manera más detallada, para que todo esté lo más controlado posible. Asimismo consensuar con los compañeros y las compañeras que también imparten esta asignatura unos cuestionarios previos al principio de cada tema.

- Tratar de comenzar siempre con el planteamiento de una pregunta de interés que guíe el desarrollo del tema y capte la atención e interés del alumnado.

- Plantear una evaluación en la que se tenga en cuenta la evaluación continua, las tareas desarrolladas en las sesiones, el examen práctico y el teórico, de modo que el control del aprendizaje no dependa de un único factor. Tratar de tener en cuenta no sólo los conocimientos técnicos y conceptuales, sino tratar de incorporar los valores, aunque esto lo tengo que pensar con más detenimiento para ver cómo. 


\section{Referencias bibliográficas}

Bain, K. (2004). Lo que hacen los mejores profesores universitarios. Valencia: Publicaciones Universidad de Valencia.

Feria-Bourrellier, A.B. (2015), “Estrategia didáctica para la enseñanza de los metabolismos fotosintéticos mediante experimentación". Comunicación en II Jornadas de Docencia Universitaria. Facultad de Ciencias de la Educación y Ciencias del Trabajo de la US. 2015

Finkel, D. (2008). Dar clases con la boca cerrada. Valencia: Publicaciones Universidad de Valencia.

Mora, F. (2017). Neuroeducación: Solo se puede aprender aquello que se ama. Madrid: Alianza Editorial.

Noguero, F.L. (2005) Metodología participativa en la enseñanza universitaria. Narcea

Porlán, R. (2017). Enseñanza Universitaria, Cómo mejorarla. Madrid: Editorial Morata.

Jornadas de Formación e Innovación Docente del Profesorado | № 1 (2018) Esta obra se distribuye con la licencia Creative Commons 